\title{
SUMMATION OF A SERIES OF PRODUCTS OF E-FUNCTIONS
}

\author{
by F. M. RAGAB
}

(Received 9th May, 1961)

1. Introductory. In previous papers $[1,2,3]$ the sums of a number of series of products of $E$-functions have been found. For the definitions and properties of the $E$-functions the reader is referred to [4, pp. 348-358]. In $\S 3$ a further series of this type is given. The proof is based on an integral of an $E$-function with respect to its parameters, to be established in $\S 2$. Similar integrals were given in [5] and [6].

The following formulae will be made use of in the proofs.

If $p \leqq q, z \neq 0,[4, \mathrm{p} .352]$

$$
\begin{gathered}
E\left(p ; \alpha_{r}: q ; \rho_{s}: z\right)=\frac{\Gamma\left(\alpha_{1}\right) \ldots \Gamma\left(\alpha_{p}\right)}{\Gamma\left(\rho_{1}\right) \ldots \Gamma\left(\rho_{q}\right)} F\left(\begin{array}{l}
p ; \alpha_{r}:-1 / z \\
q ; \rho_{s}
\end{array}\right) . \\
\text { If } R\left(\rho_{q+1}\right)>R\left(\alpha_{p+1}\right)>0,[4, \mathrm{p} .395] \\
\int_{0}^{1} \lambda^{\alpha_{p+1}-1}(1-\lambda)^{\rho_{q+1}-\alpha_{p+1}-1} E\left(p ; \alpha_{r}: q ; \rho_{s}: z / \lambda\right) d \lambda \\
=\Gamma\left(\rho_{q+1}-\alpha_{p+1}\right) E\left(p+1 ; \alpha_{r}: q+1 ; \rho_{s}: z\right) .
\end{gathered}
$$

If $R\left(\alpha_{p+1}\right)>0,[4, p .394]$

$$
\int_{0}^{\infty} e^{-\lambda} \lambda^{\alpha_{p+1}-1} E\left(p ; \alpha_{r}: q ; \rho_{s}: z / \lambda\right) d \lambda=E\left(p+1 ; \alpha_{r}: q ; \rho_{s}: z\right) .
$$

If $|z|<1,[7$, p. 100]

$$
F\left(\begin{array}{l}
\alpha, \beta: \\
\alpha+\beta+\frac{1}{2}
\end{array}\right) F\left(\begin{array}{l}
\frac{1}{2}-\alpha, \frac{1}{2}-\beta: z \\
\frac{3}{2}-\alpha-\beta
\end{array}\right)=F\left(\begin{array}{l}
\alpha-\beta+\frac{1}{2}, \beta-\alpha+\frac{1}{2}, \frac{1}{2}: z \\
\alpha+\beta+\frac{1}{2}, \frac{3}{2}-\alpha-\beta
\end{array}\right) .
$$

If $|\operatorname{amp} z|<\pi,[4$, p. 374]

$$
E\left(p ; \alpha_{r}: q ; \rho_{s}: z\right)=\frac{1}{2 \pi i} \int \frac{\Gamma(\zeta) \Pi \Gamma\left(\alpha_{r}-\zeta\right)}{\Pi \Gamma\left(\rho_{s}-\zeta\right)} z^{\zeta} d \zeta
$$

where the contour of integration is taken up the $\eta$-axis with loops, if necessary, to ensure that the pole at the origin lies to the left and the poles at $\alpha_{1}, \alpha_{2}, \ldots, \alpha_{p}$ to the right of the contour. Zero and negative integral values of the parameters are excluded. If $p<q+1$ the contour is bent to the left at both ends. When $p>q+1$ the formula is valid for $\mid$ amp $z \mid<\frac{1}{2}(p-q+1) \pi$.

By applying (1) to (4) it can be deduced that

$$
\begin{aligned}
E\left(\begin{array}{c}
\alpha, \beta: \\
\alpha+\beta+\frac{1}{2}
\end{array}\right) E\left(\begin{array}{c}
\frac{1}{2}-\alpha, \frac{1}{2}-\beta: z \\
\frac{3}{2}-\alpha-\beta
\end{array}\right)=\cos (\alpha-\beta) \pi & \\
& \times \pi^{-3 / 2} \Gamma(\alpha) \Gamma(\beta) \Gamma\left(\frac{1}{2}-\alpha\right) \Gamma\left(\frac{1}{2}-\beta\right) E\left(\begin{array}{c}
\alpha-\beta+\frac{1}{2}, \beta-\alpha+\frac{1}{2}, \frac{1}{2}: z \\
\alpha+\beta+\frac{1}{2}, \frac{3}{2}-\alpha-\beta
\end{array}\right) .
\end{aligned}
$$


Finally, $[4$, p. 351]

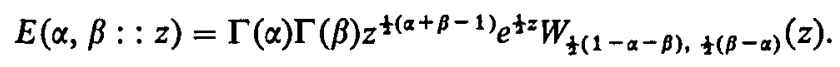

2. Integration of an E-function with respect to its parameters. The formula to be proved is

$$
\begin{aligned}
& \frac{1}{2 \pi i} \int \frac{\Gamma(\zeta) \Gamma(\alpha-\zeta) \Gamma(\beta-\zeta)}{\Gamma\left(\alpha+\beta+\frac{1}{2}-\zeta\right)} z^{\zeta} E\left(\begin{array}{l}
\frac{1}{2}-\alpha, \frac{1}{2}-\beta, \alpha_{1}-\zeta, \ldots, \alpha_{p}-\zeta: z \\
\frac{3}{2}-\alpha-\beta, \rho_{1}-\zeta, \ldots, \rho_{q}-\zeta
\end{array}\right) d \zeta \\
& \quad=\pi^{-3 / 2} \cos (\alpha-\beta) \pi \Gamma(\alpha) \Gamma(\beta) \Gamma\left(\frac{1}{2}-\alpha\right) \Gamma\left(\frac{1}{2}-\beta\right) E\left(\begin{array}{l}
\frac{1}{2}, \alpha-\beta+\frac{1}{2}, \beta-\alpha+\frac{1}{2}, \alpha_{1}, \ldots, \alpha_{p}: z \\
\alpha+\beta+\frac{1}{2}, \frac{3}{2}-\alpha-\beta, \rho_{1}, \ldots, \rho_{q}
\end{array}\right),
\end{aligned}
$$

where $p \geqq q,|\operatorname{amp} z|<\frac{1}{2}(p-q+2) \pi, R\left(\rho_{n}-\alpha_{n}\right)>0(n=1,2, \ldots, q), R\left(\alpha_{n}\right)>0(n=1,2, \ldots, p)$, $\alpha$ and $\beta$ being such that the $E$-functions exist. The contour of integration is the same as in (5) with loops, if necessary, to ensure that $\alpha$ and $\beta$ are to the right of the contour.

From (2) and (3) it follows that the left-hand side of (8) is equal to

$$
\begin{aligned}
\frac{1}{2 \pi i} \int \frac{\Gamma(\zeta) \Gamma(\alpha-\zeta) \Gamma(\beta-\zeta)}{\Gamma\left(\alpha+\beta+\frac{1}{2}-\zeta\right)} z^{\zeta}\left[\prod_{n=1}^{q}\right. & \left.\Gamma\left(\rho_{n}-\alpha_{n}\right)\right]^{-1} \prod_{n=1}^{q} \int_{0}^{1} \lambda_{n}^{\alpha_{n}-\zeta-1}\left(1-\lambda_{n}\right)^{\rho_{n}-\alpha_{n}-1} d \lambda_{n} \\
& \times \prod_{n=q+1}^{p} \int_{0}^{\infty} e^{-\lambda_{n} \lambda_{n}^{\alpha_{n}-\zeta-1} d \lambda_{n} E\left(\frac{1}{2}-\alpha, \frac{1}{2}-\beta: z / \lambda_{1} \lambda_{2} \ldots \lambda_{p}\right) d \zeta .}
\end{aligned}
$$

Here change the order of the factors and get

$$
\begin{aligned}
& {\left[\prod_{n=1}^{q} \Gamma\left(\rho_{n}-\alpha_{n}\right)\right]^{-1} \prod_{n=1}^{q} \int_{0}^{1} \lambda_{n}^{\alpha_{n}-1}\left(1-\lambda_{n}\right)^{\rho_{n}-\alpha_{n}-1} d \lambda_{n}} \\
& \times \prod_{n=q+1}^{p-1} \int_{0}^{\infty} e^{-\lambda_{n} \lambda_{n}^{\alpha_{n}-1}} d \lambda_{n} \int_{0}^{\infty} e^{-\lambda_{p}} \lambda_{p}^{\alpha_{p}-1} E\left(\begin{array}{l}
\frac{1}{2}-\alpha, \frac{1}{2}-\beta: z /\left(\lambda_{1} \ldots \lambda_{p}\right) \\
\frac{3}{2}-\alpha-\beta
\end{array}\right) d \lambda_{p} \\
& \times \frac{1}{2 \pi i} \int \frac{\Gamma(\zeta) \Gamma(\alpha-\zeta) \Gamma(\beta-\zeta)}{\Gamma\left(\alpha+\beta+\frac{1}{2}-\zeta\right)}\left(\frac{z}{\lambda_{1} \ldots \lambda_{p}}\right)^{\zeta} d \zeta .
\end{aligned}
$$

On substituting from (5) for the last integral the expression becomes

$$
\begin{aligned}
& {\left[\prod_{n=1}^{q} \Gamma\left(\rho_{n}-\alpha_{n}\right)\right]^{-1} \prod_{n=1}^{q} \int_{0}^{1} \lambda_{n}^{\alpha_{n}-1}\left(1-\lambda_{n}\right)^{\rho_{n}-\alpha_{n}-1} d \lambda_{n} \prod_{n=q+1}^{p-1} \int_{0}^{\infty} e^{-\lambda_{n}} \lambda_{n}^{\alpha_{n}-1} d \lambda_{n}} \\
& \times \int_{0}^{\infty} e^{-\lambda_{p} \lambda_{p}^{\alpha_{p}-1} E} E\left(\begin{array}{l}
\alpha, \beta \quad: z / \lambda_{1} \ldots \lambda_{p} \\
\alpha+\beta+\frac{1}{2}
\end{array}\right) E\left(\begin{array}{l}
\frac{1}{2}-\alpha, \frac{1}{2}-\beta: z / \lambda_{1} \ldots \lambda_{p} \\
\frac{3}{2}-\alpha-\beta
\end{array}\right) d \lambda_{p} .
\end{aligned}
$$

Now substitute from (6) and, on integrating, using (2) and (3), formula (8) is obtained. The restrictions on the $\rho$ 's can be removed as the paths of integration from 0 to 1 can be replaced by contours which start from 0 , pass round the point 1 and return to 0 . 
3. A series of products of E-functions. It will now be shown that the formula (8) can be applied to obtain the series

$$
\begin{aligned}
& \sum_{r=0}^{\infty} \frac{z^{-2 r}}{r !(\gamma ; r)} E\left(\begin{array}{l}
\alpha+r, \beta+r, \gamma+r: z \\
\alpha+\beta+\frac{1}{2}+r
\end{array}\right) E\left(\begin{array}{l}
\frac{1}{2}-\alpha+r, \frac{1}{2}-\beta+r, \gamma+r: z \\
\frac{3}{2}-\alpha-\beta+r
\end{array}\right) \\
& \quad=\pi^{-3 / 2} \cos (\alpha-\beta) \pi \Gamma(\alpha) \Gamma(\beta) \Gamma\left(\frac{1}{2}-\alpha\right) \Gamma\left(\frac{1}{2}-\beta\right) \Gamma(\gamma) E\left(\begin{array}{l}
\alpha-\beta+\frac{1}{2}, \beta-\alpha+\frac{1}{2}, \gamma, \frac{1}{2}: z \\
\alpha+\beta+\frac{1}{2}, \frac{3}{2}-\alpha-\beta
\end{array}\right),
\end{aligned}
$$

where $|\operatorname{amp} z|<\frac{3}{2} \pi, R(\gamma)>0,0<R(\alpha)<\frac{1}{2}, 0<R(\beta)<\frac{1}{2}$.

To prove this substitute from (5) for the $E$-functions on the left of (9) and get

$$
\begin{aligned}
& \sum_{r=0}^{\infty} \frac{z^{-2 r}}{r !(\gamma ; r)} \frac{1}{2 \pi i} \int \frac{\Gamma(\zeta) \Gamma(\alpha+r-\zeta) \Gamma(\beta+r-\zeta) \Gamma(\gamma+r-\zeta)}{\Gamma\left(\alpha+\beta+\frac{1}{2}+r-\zeta\right)} z^{\zeta} d \zeta \\
& \times \frac{1}{2 \pi i} \int \frac{\Gamma(Z) \Gamma\left(\frac{1}{2}-\alpha+r-Z\right) \Gamma\left(\frac{1}{2}-\beta+r-Z\right) \Gamma(\gamma+r-Z)}{\Gamma\left(\frac{3}{2}-\alpha-\beta+r-Z\right)} z^{Z} d Z .
\end{aligned}
$$

Here replace $\zeta$ and $Z$ by $\zeta+r$ and $Z+r$, and interchange the order of summation and integration, so getting

$$
\begin{aligned}
\frac{1}{2 \pi i} \int \frac{\Gamma(\zeta) \Gamma(\alpha-\zeta) \Gamma(\beta-\zeta) \Gamma(\gamma-\zeta)}{\Gamma\left(\alpha+\beta+\frac{1}{2}-\zeta\right)} & z^{\zeta} d \zeta \\
& \times \frac{1}{2 \pi i} \int \frac{\Gamma(Z) \Gamma\left(\frac{1}{2}-\alpha-Z\right) \Gamma\left(\frac{1}{2}-\beta-Z\right) \Gamma(\gamma-Z)}{\Gamma\left(\frac{3}{2}-\alpha-\beta-Z\right)} z F\left(\begin{array}{c}
\zeta, Z ; 1 \\
\gamma
\end{array}\right) d Z .
\end{aligned}
$$

On applying Gauss's theorem this becomes

$$
\frac{\Gamma(\gamma)}{2 \pi i} \int \frac{\Gamma(\zeta) \Gamma(\alpha-\zeta) \Gamma(\beta-\zeta)}{\Gamma\left(\alpha+\beta+\frac{1}{2}-\zeta\right)} z^{\zeta} E\left(\begin{array}{c}
\frac{1}{2}-\alpha, \frac{1}{2}-\beta, \gamma-\zeta: z \\
\frac{3}{2}-\alpha-\beta
\end{array}\right) d \zeta
$$

and, from (8), with $p=1, q=0$, the result follows.

Note. On replacing $\gamma$ in (9) by $\alpha+\beta+\frac{1}{2}$ or $\frac{3}{2}-\alpha-\beta$ and applying (7), series involving Whittaker functions can be obtained.

\section{REFERENCES}

1. F. M. Ragab, Expansion of an $E$-function in a series of products of $E$-functions, Proc. Glasgow Math. Assoc. 3 (1958), 194-195.

2. F. M. Ragab, Expansions for products of two Whittaker functions, Div. Electromag. Res., Inst. Math. Sci., New York Univ., Res. Rep. No. BR-23 (1957).

3. F. M. Ragab, An expansion involving confluent hypergeometric functions, Nieuw Arch. Wisk. (3) 6 (1958), 52-54.

4. T. M. MacRobert, Functions of a complex variable, 4th edn. (London, 1954).

5. F. M. Ragab, Integration of $E$-functions and related functions with respect to their parameters, Nederl. Akad. Wetensch. Proc. Ser. A 61 (1958), 335-340.

6. F. M. Ragab, Integration of $E$-functions with regard to their parameters, Proc. Glasgow Math. Assoc. 3 (1957), 94-98.

7. W. N. Bailey, Generalized hypergeometric series (Cambridge, 1935).

Cairo University

CAIRO 